\title{
Analytic estimates and topological properties of the weak stability boundary
}

\author{
Marta Ceccaroni · James Biggs · Luca \\ Biasco
}

\begin{abstract}
The weak stability boundary (WSB) is the transition region of the phase space where the change from gravitational escape to ballistic capture occurs. Studies on this complicated region of chaotic motion aim to investigate its unique, fuel saving properties to enlarge the frontiers of low energy transfers. This "fuzzy stability" region is characterized by highly sensitive motion, and any analysis of it has been carried out almost exclusively using numerical methods.

On the contrary this paper presents, for the planar circular restricted 3 body problem (PCR3BP), 1) an analytic definition of the WSB which is coherent with the known algorithmic definitions; 2) a precise description of the topology of the WSB; 3) analytic estimates on the "stable region" (nearby the smaller primary) whose boundary is, by definition, the WSB.
\end{abstract}

Keywords Weak Stability boundary · Restricted Three Body Problem

\section{Introduction and main results}

In January 1990 Japan's ISAS Institute launched a pair of small spacecraft linked together into an elliptic Earth orbit. The smaller one, called MUSES-B, detached and lost communication connections; it was supposed to go to the Moon and into lunar orbit using a Hohmann transfer. The larger craft, MUSES-A, still orbiting the Earth was meant to send and collect communications to and from MUSES-B, and perform

Marta Ceccaroni

Advanced Space Concepts Laboratory

University of Strathclyde, Glasgow, UK

E-mail: marta.ceccaroni@strath.ac.uk

James Biggs

Advanced Space Concepts Laboratory

University of Strathclyde, Glasgow, UK

E-mail: james.biggs@strath.ac.uk

Luca Biasco

University of Roma Tre, Rome, IT

E-mail: biasco@mat.uniroma3.it 
scientific experiments while in Earth orbit. It was then desired by the ISAS Institute to try to get MUSES-A to the Moon as a replacement for MUSES-B, and into lunar orbit with a desired lunar periapsis radius at capture $r$ equal to $r_{M o o n}+100 K m$. MUSES-A had a very small $\Delta V$ capability of approximately 100 meters per second $(\mathrm{m} / \mathrm{s})$, far less than what is necessary to be placed into lunar orbit using an Hohmann transfer as it was never designed to go to the Moon. A solution was found by Belbruno and Miller (1993) at the Jet Propulsion Laboratory in June 1990 to enable MUSES-A, renamed Hiten, to reach the Moon on a ballistic capture transfer to the region $\mathcal{W}$, thus named lunar Weak Stability Boundary (WSB). This transfer rescued the Japanese lunar mission; without it, there was not enough $\Delta V$ to get Hiten to the Moon by any other means.

Since the Hiten mission the low energy interplanetary transfers have been widely studied (see for example: Belbruno (1987), Belbruno (1990), Belbruno (2004) and Topputo (2007)), particularly, arousing the interest in designing lunar trajectories (Koon et al. (2001), Gómez et al (1993), Romagnoli and Circi (2009) and Mingotti et al. (2009)) and other systems (Topputo and Belbruno, 2009). Many different ways have been proposed for designing them (Belbruno (1987), Carrico and Belbruno (2006), Bello-Mora et al. (2000), Belbruno (1997), Gómez et al. (2004)). All of these techniques for " ballistic capture transfers" used the WSB. Moreover, this zone has the unique property that a spacecraft entering it is automatically captured in an elliptical orbit without needing to reduce its velocity with the use of rockets.

Roughly speaking the WSB is described as "a generalization of the Lagrange points and a complicated region surrounding the Moon" Belbruno and Miller (1993), "a region in phase space supporting a special type of chaotic motion for special choices of elliptic initial conditions with respect to $m_{2}$ " Belbruno (2004), "a transition region between the gravitational capture and escape from the Moon in the phase space" Topputo et al. (2008).

More precisely in Belbruno (2004) the author suggested an algorithmic definition of WSB and presented its geometry for the Earth-Moon system in the planar case, in terms of eccentricity, periselenium altitude and Jacobi constant of the spacecraft osculating initial orbit. However García and Gómez (2007) spotted some lack of accuracy in the definition given by Belbruno, thus providing a new algorithmic definition which addressed all the comments on the previous one, and gave numerical evidence that the shape of the WSB arises from their definition.

\subsection{Main results}

In this paper, starting from the algorithmic definitions cited above, an analytical definition of the WSB (see Definition 9) is given for the planar, circular, restricted three-body problem (PCR3BP). We consider the motions of a massless body (restricted problem) subject to the gravitational attractions of two massive primaries revolving on planar circular orbits (circular problem), around their common center of mass. The massless body is assumed to move in the same plane as the primaries (planar problem). For simplicity of exposition we will call "Spacecraft", "Earth" and "Moon", the massless body, the larger primary and the smaller primary respectively. However we stress that our analysis is general and holds for every PCR3BP.

In the rotating reference frame in which the Moon is at the origin and the Earth is 
at rest at distance 1 (see the reference frame F2 on page 6), we consider a half-line $l$ emanating from the Moon with inclination of an angle $\theta$ with respect to the line joining the Earth and the Moon. Given an eccentricity $e \in[0,1]$ and a distance $r>0$ we consider an $(r, e, \theta)$ - test orbit (see Definition 4), namely an orbit starting from $l$ at a distance $r$ from the Moon, with initial velocity such that, the starting point is at the periselenium (or aposelenium) of the "osculating ellipse" with eccentricity $e$. The "osculating ellipse" is defined as the ellipse that the Spacecraft would make neglecting the influence of the Earth.

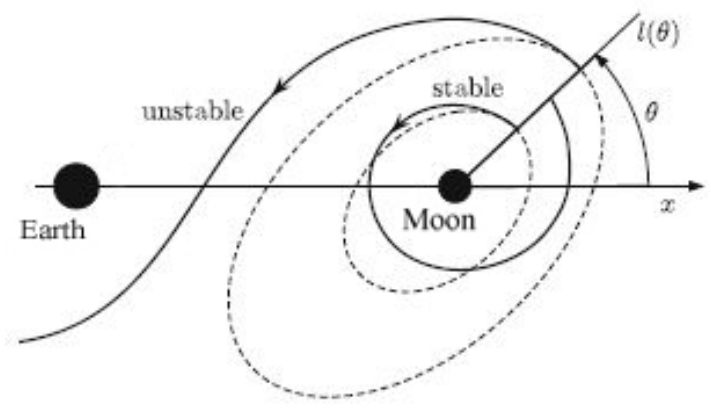

Fig. 1 Stable and unstable test-orbits

A $(r, e, \theta)$-test orbit is "stable" (see Definition 5) if it makes a full circle around the Moon without going around the Earth or crashing into the Earth and returns to a point of $l$ with $H_{M} \leq 0$, where $H_{M}$ is defined as the two-body energy of the system Spacecraft-Moon (see Definition 1); moreover, following Topputo (2007), we also require it to come back on $l$ with strictly positive angular velocity ("transversality" condition). We call $S(e, \theta) \subset \mathbb{R}^{+}$(see (16)) the set of $r$ 's such that the $(r, e, \theta)$-test orbit is stable and satisfies the transversality condition. Then we set $\mathcal{W}(e, \theta):=\partial S(e, \theta)$ and finally define the WSB as

$$
\mathcal{W}:=\left\{(r, e, \theta) \in \mathbb{R}^{+} \times[0,1] \times S^{1} \text { s.t. } r \in \mathcal{W}(e, \theta)\right\}
$$

(see Definition 9).

We note that, thanks to the topological characterization of the WSB (see Proposition 1 below) we are able to show that our analytic definition of WSB and the algorithmic one given in García and Gómez (2007) are coherent and give rise to the same picture for the WSB, see Remark 2.

Using our definition of the WSB, in particular by the transversality condition and by the fact that the Hamiltonian of the PCR3BP is an analytic function, we are able to show a precise description of the topology of the set of stable orbits and therefore of the WSB. Such result is stated in the following proposition which will be proved in Section 3

Proposition 1 For every $(e, \theta) \in(0,1) \times S^{1}, S(e, \theta)$ is a bounded set formed by at most a countable disjoint union of open intervals plus at most a countable set of points. Moreover there exists $r^{*}(e)>0$ such that $S(e, \theta) \supset\left(0, r^{*}(e)\right)$. 
Then we study the stable zone $S(e, \theta)$, giving an analytic estimate from below on $r^{*}(e)$, see Corollary 1.

For low values of the eccentricity good stability estimates can be obtained working with the usual polar coordinates (see the reference frame F2 on page 6). However, in view of the applications (i.e. a comparison with the results in Belbruno (2004)), one is interested in considering eccentricities close to 1 . In this case the stability estimates deteriorate, moreover polar coordinates are not convenient for analytic estimates anymore. Thus we consider the symplectic Delaunay coordinates and we rewrite the WSB problem in such coordinates (see Section 4). It results that the Delaunay coordinates are suitable for analytic estimates in the large eccentricity regime. Indeed we perform some analytic estimates on the Hamiltonian vector field in Delaunay variables (see Lemma 2), and we use the assumption that, if the spacecraft starts quite close to the Moon, then the "mean anomaly" is a so called "fast angle". Such estimates allow us to state the Stability Proposition 2, which gives the desired estimate from below on $r^{*}(e)$ contained in Corollary 1.

As an example we explicitly evaluate the value of $r^{*}(e)$ when $e=0.95$, which is the initial eccentricity used by Belbruno in the Hiten mission (see p.145 of Belbruno (2004)). It results that $r^{*}(0.95)$ is about $1 / 7$ of Belbruno's escaping radius considered for the Hiten's mission (see Subsection 5.1). In order to test the validity of the analytical result we also perform a numerical estimation of $r^{*}(0.95)$ which result to be about $1 / 3$ of Hiten's escaping ray.

The paper is organized as follows.

In Section 2 we recall the definitions of the WSB given by Belbruno (see Definition 7) and García and Gómez (2007). (see Definition 8). Then we also propose our analytic definition (see Definition 9), making some comparison with the other definitions.

The topology of the WSB is studied in Section 3, where Proposition 1 is proved.

A complete description of the WSB problem in Delaunay variables is given in Section 4.

In Section 5 we prove the Stability Proposition 2, whose immediate consequence is Corollary 1 . Moreover we explicitly evaluate $r^{*}(0.95)$.

Finally in the Appendix we prove the technical Lemma 2 used in Section 5.

\section{Various definitions of the WSB}

Three main definitions of Weak Stability Boundaries are introduced in this section. The first is the algorithmic definition given by Belbruno (see for example Belbruno (2004)). The second is the modified version given in García and Gómez (2007) which provide a second, more complete, definition of the WSB set, although still algorithmic. Finally the latter is a new, analytic definition of the WSB, followed by a topological analysis of it and a comparison with the previous definitions, which represents one of the novelties of this paper.

All these definitions are based on the concept of "ballistic capture" which is also presented, following the definition in Belbruno (2004) (see Definition 2).

Moreover, as the definition of Ballistic Capture is based on monitoring the sign of Kepler's energy function of the Moon-Spacecraft system, a definition of such Two Body Keplerian Energy is also given (see Definition 1), for different reference frames, equiv- 
alent to the one given in Belbruno (2004).

2.1 The Hamiltonian of the PCR3BP

We normalize

$$
\begin{aligned}
& M=\text { mass of the Moon, } \quad 1-M=\text { mass of the Earth; } \\
& \text { Earth }- \text { Moon distance }=1, \quad \text { angular velocity }=1 .
\end{aligned}
$$

F0) In a fixed inertial frame F0 with the origin in the center of mass of the MoonEarth system (recall that the Spacecraft has zero mass) the motions of the Moon and the Earth are, respectively,

$$
(M-1) c(t) \text { and } M c(t) \text { where } c(t):=(\cos (t-\pi), \sin (t-\pi))
$$

(the Moon and the Earth starting at $1-M,-M$ for $t=0$, respectively).

Denoting by $s \in \mathbb{R}^{2}$ the position of the massless body, i.e. the Spacecraft $(S)$, the equation of motions for the orbit $s(t)$ of $S$ is given by

$$
\ddot{s}=-M \frac{s-(M-1) c(t)}{|s-(M-1) c(t)|^{3}}-(1-M) \frac{s-M c(t)}{|s-M c(t)|^{3}} .
$$

F1) Let us now pass to a selenocentric (non inertial) frame F1 with the Moon at the origin and the Earth moving with angular velocity 1 on a unitary circle around the Moon. Assuming that at the time $t=0$ the Earth is in $(-1,0)$, the orbit of the Earth is $c(t)$. the new coordinates of $S$ are given by

$$
x=s-(M-1) c(t) .
$$

Then the equation of motions is

$$
\ddot{x}=\ddot{s}+(M-1) c(t)=-M \frac{x}{|x|^{3}}-(1-M) \frac{x-c(t)}{|x-c(t)|^{3}}+(M-1) c(t) .
$$

Equation (1) is equivalent to the Hamiltonian system

$$
\dot{X}=-\partial_{x} H_{1}, \quad \dot{x}=\partial_{X} H_{1}
$$

with Hamiltonian

$$
H_{1}(X, x ; t):=\frac{|X|^{2}}{2}-\frac{M}{|x|}-\frac{1-M}{|x-c(t)|}+(1-M) c(t) \cdot x
$$

in the conjugated variables $X, x \in \mathbb{R}^{2}$ with symplectic form $d X \wedge d x$.

Note that $X=\dot{x}$ is the velocity.

Definition 1 (Two-Body Kepler energy) In the selenocentric frame F1 defined above with conjugate variables velocity-position $(X, x)$ we define the two-Body Kepler energy of $S$ with respect to the Moon:

$$
H_{M}(X, x):=\frac{|X|^{2}}{2}-\frac{M}{|x|} .
$$


Heuristically $H_{M}$ can be seen has the Hamiltonian (in the selenocentric frame) due to the Moon only, namely when one neglects the influence of the Earth (formally setting $1-M=0$ in $H_{1}$, see $\left.(2)\right)$.

Passing to polar coordinates $(R, \Phi, r, \varphi)$ by the symplectic transformation

$$
x=r(\cos \varphi, \sin \varphi), \quad X=\left(R \cos \varphi-\frac{\Phi}{r} \sin \varphi, R \sin \varphi+\frac{\Phi}{r} \cos \varphi\right)
$$

the Hamiltonian $H_{1}$ in (2) is transformed into

$$
\begin{aligned}
& H_{2}(R, \Phi, r, \varphi ; t)= \\
& \quad \frac{1}{2}\left(R^{2}+\frac{\Phi^{2}}{r^{2}}\right)-\frac{M}{r}-\frac{1-M}{\sqrt{r^{2}+1-2 r \cos (\varphi-t+\pi)}}+(1-M) r \cos (\varphi-t+\pi) .
\end{aligned}
$$

Moreover

$$
H_{M}(R, \Phi, r, \varphi)=\frac{1}{2}\left(R^{2}+\frac{\Phi^{2}}{r^{2}}\right)-\frac{M}{r}
$$

F2) Let us consider the anti-clockwise rotating frame F2, with angular velocity 1 , in which both the Moon and the Earth are at rest; with the Moon at the origin and the Earth at $(-1,0)$. Let us denote by $(r, \vartheta)$ the polar coordinates in this rotating frame where $\theta$ is the angle measured from the horizontal line joining the Earth and the Moon. After the time-dependent canonical transformation

$$
\vartheta=\varphi-t, \quad \Theta=\Phi
$$

the Hamiltonian $\mathrm{H}_{2}$ in (4) is transformed into the autonomous Hamiltonian

$$
\begin{aligned}
& H_{3}(R, \Theta, r, \vartheta)= \\
& \frac{1}{2}\left(R^{2}+\frac{\Theta^{2}}{r^{2}}\right)-\frac{M}{r}-\Theta-\frac{1-M}{\sqrt{r^{2}+1-2 r \cos (\vartheta+\pi)}}+(1-M) r \cos (\vartheta+\pi) .
\end{aligned}
$$

Moreover

$$
H_{M}(R, \Theta, r, \vartheta)=\frac{1}{2}\left(R^{2}+\frac{\Theta^{2}}{r^{2}}\right)-\frac{M}{r}
$$

\subsection{Belbruno's definition of WSB}

Let us consider a solution $\psi(t)$ of the above Hamiltonians. Note that it is not important to specify if we are considering $H_{1}, H_{2}$ or $H_{3}$ since they are related by symplectic changes of variables. Obviously $H_{M}$ must be intended in the same variables of the corresponding Hamiltonian.

Definition 2 (Ballistic Capture)

Given $0<t_{1}<\infty$. A Spacecraft is said to be ballistically captured by the Moon in $t=t_{1}$ if

$$
H_{M}\left(\psi\left(t_{1}\right)\right) \leq 0 \text {. }
$$


Definition 3 (Osculating ellipse) In the selenocentric frame F1 the the osculating ellipse, is the orbit of the two-body problem Moon-spacecraft (neglecting the Earth) made by the spacecraft around the Moon, with negative energy. Where the moon is set in one of the foci of the ellipse. In other words we are considering the motions of the Hamiltonian $H_{M}$ when the energy is negative.

We recall the relation between the orbital elements of the osculating ellipse (see, e.g., Celletti and Chierchia (2007) for details): the eccentricity $e$, the energy $E$, the major semi-axis $a$, the minimal $r_{\min }$ and maximal $r_{\max }$ distance from the Moon (periselenium and aposelenium respectively)

$$
E=-\frac{M}{2 a}, \quad r_{\min }=a(1-e), \quad r_{\max }=a(1+e) .
$$

Definition $4\left(\left(r_{0}, e_{0}, \vartheta_{0}\right)\right.$-Test orbit) In $\mathbf{F} 1$ consider radial line $l$ emanating from the Moon and inclined of an angle $\vartheta_{0} \in S^{1}$ with respect to the axis joining the Earth and the Moon (see Fig.1). Then the $\left(\left(r_{0}, e_{0}, \vartheta_{0}\right)\right.$-Test orbit) is the trajectory of the spacecraft which at time $t=0$ :

- departs from the line $l$ at a distance $r_{0}>0$ from the Moon

- is at the periselenium or at the aposelenium of the osculating ellipse, i.e. its initial velocity vector is normal to the line $l$, pointing in the direct or retrograde direction

- with a fixed initial eccentricity ${ }^{1} e_{0} \in[0,1]$.

Therefore the initial conditions in polar coordinates are (recalling (5) and (10)):

$$
R(0)=0, \quad \Phi(0)= \pm \sqrt{M r_{0}(1 \pm e)}, \quad r(0)=r_{0}, \quad \varphi(0)=\vartheta_{0},
$$

where in $\Phi(0)$ the first \pm is + in the direct direction and - in the retrograde one and the second \pm depends if one is at the periselenium or at the aposelenium.

Analogously, in F2, consider radial line $l$ emanating from the Moon and inclined of an angle $\vartheta_{0}$ with respect to the horizontal axis (see Fig.1). The same orbit as above has, in the rotating frame $\mathbf{F 2}$, the initial data (recall (6) and since $t=0$ ):

$$
R(0)=0, \quad \Theta(0)= \pm \sqrt{M r_{0}(1 \pm e)}, \quad r(0)=r_{0}, \quad \vartheta(0)=\vartheta_{0},
$$

Definition 5 (Stability)

A test orbit is said to be stable about the Moon if, after leaving $l$, makes a full cycle about the Moon in a time $T>0$ without going around the Earth or crashing into the Earth and returns to a point on $l$ with $H_{M}(\psi(T)) \leq 0$.

Definition 6 (Instability)

A test orbit is said to be unstable about the Moon if:

- it performs a full cycle around the Moon without going around the Earth and returns to a point on $l$ with $H_{M}>0$

- the Spacecraft moves away from the Moon towards the Earth and makes a full cycle about it or collides with it.

The stability definition above corresponds to the ballistic capture with respect to the Moon given in Definition 2 at time both 0 and $T$ ("ballistic capture transfer"). The first condition of instability is called ballistic escape from the Moon, while the second is called primary interchange escape.

\footnotetext{
1 This implies that the two body Kepler energy is negative.
} 
For every fixed $(e, \theta) \in[0,1] \times S^{1}$ Belbruno claims (Belbruno, 2004) that it is possible to find numerically a finite distance $r^{\prime}=r^{\prime}(e, \theta)>0$ from the Moon such that a $(r, e, \theta)$-test orbit is

$$
\text { stable if } r<r^{\prime}(e, \theta), \quad \text { unstable if } r>r^{\prime}(e, \theta) .
$$

Definition 7 (Belbruno's WSB)

$$
\mathcal{W}:=\left\{(r, e, \theta) \in \mathbb{R}^{+} \times[0,1] \times S^{1} \text { s.t. } r=r^{\prime}(e, \theta)\right\} .
$$

Note that $\mathcal{W}$ is a two dimensional stability transition region of position and velocity, which has two components corresponding to the direct and retrograde motions.

\subsection{García-Gómez definition of WSB}

García and Gómez (2007) stress some lack of accuracy in Belbruno's definitions. One regards the definition of unstable orbits, and the other the definition of the radius of change of stability $r^{\prime}(\theta, e)$.

More precisely, the latter arises from noticing that Definition 7 is not exhaustive of the complementary set of the Stable orbits as given in Definition 6. Therefore a more general definition of instability is adapted in García and Gómez (2007), where is defined as unstable any orbit that does not fulfill the Stability criterion. On the other hand, they highlight that it is not clear how, for fixed values of $\theta$ and $e$ there exist a finite distance $r^{\prime}(\theta, e)$ defining the boundary between stable and unstable orbits. In fact it is shown in the paper that for each fixed inclination and eccentricity there are many changes from stability to instability and that the set of the stable points recalls a Cantor set.

Then García and Gómez (2007) proposed an extended definition of WSB:

Definition 8 ( García-Gómez WSB) $\forall(e, \theta) \in[0,2 \pi] \times[0,1]$ there exist a finite number of points (up to a certain precision) $r_{1}^{*}=0, r_{2}^{*}=r_{2}^{*}(e, \theta), \ldots, r_{2 n}^{*}=r_{2 n(e, \theta)}^{*}$ such that if $r$ belongs to

$$
S^{*}(e, \theta):=\bigcup_{1 \leq j \leq n}\left[r_{2 j-1}^{*}, r_{2 j}^{*}\right]
$$

the motion of a $(r, e, \theta)$-test orbit is stable according to Definition 5 otherwise it is unstable. Then the WSB is defined as:

$\mathcal{W}:=\left\{(r, e, \theta) \in \mathbb{R}^{+} \times[0,1] \times S^{1}\right.$ s.t. $\left.r \in \mathcal{W}(e, \theta)\right\}$ where $\mathcal{W}(e, \theta):=\partial S^{*}(e, \theta) \subset \mathbb{R}^{+}$.

The number of points $r_{i}^{*}$ and their values, varies with the values of $\theta$ and $e$, as well as with the precision of computation.

As $r_{1}^{*}=0$, the radius $r_{2}^{*}(e, \theta)$, for each fixed $\theta$ and $e$, represents the bigger radius such that $\forall 0<r<r_{2}^{*}(e, \theta)$, a $(r, e, \theta)$-test orbit is stable. Therefore, $\forall \theta$, $e$ fixed, $r_{2}^{*}(e, \theta)$ could be seen as a lower limit of the WSB. However, as the stability/instability of an orbit is determined in a purely numerical way, all these properties would be true only limited to a certain precision ${ }^{2}$, which means that nothing prevent an unstable

\footnotetext{
2 Note that Belbruno's definition of WSB is affected by the same dependence from the accuracy of the numerical evaluation
} 
test orbit to be found in the stable interval $\left(0, r_{2}^{*}(e, \theta)\right]$, once the precision has been increased. In Section 5 we will give analytic estimates on $r_{2}^{*}(e, \theta)$ (i.e. with "infinite" precision).

\subsection{Our definition of WSB}

Remark 1 In the following we consider the component of the WSB generated by direct motions; analogous consideration for the retrograde motions are possible (leading to better stability estimates in Section 5).

Interesting topological considerations on the WSB are possible if we add to the definition of stability given in Definition 5 the request that the angular velocity $\dot{\vartheta}(T)$ of the test orbit (see Definition 4) at the time $T>0$ of first return to the line $l$ (after making a full cycle around the Moon) is strictly positive, namely $\dot{\vartheta}(T)>0$. This "transversality" condition is (sometimes) implicitly assumed by Belbruno and García and Gómez (2007) and explicitly by Topputo (2007).

For $(e, \theta) \in[0,1] \times S^{1}$ we set

$S=S(e, \theta):=\{r>0$ s.t. the $(r, e, \theta)-$ test orbit is stable according to Definition 5

and $\dot{\vartheta}(T)>0\}$.

Then we can give our definition of the WSB:

\section{Definition 9}

$\mathcal{W}:=\left\{(r, e, \theta) \in \mathbb{R}^{+} \times[0,1] \times S^{1}\right.$ s.t. $\left.r \in \mathcal{W}(e, \theta)\right\} \quad$ where $\mathcal{W}(e, \theta):=\partial S(e, \theta) \subset \mathbb{R}^{+}$.

We now investigate the relation between our analytic definition of the WSB and the previous ones, that are algorithmic in nature.

Given a $\delta>0$ we define a subset $S_{\delta}(e, \theta)$ of $S(e, \theta)$, which is a " $\delta$-approximation" of $S(e, \theta)$. By Proposition $1 S(e, \theta)$ is the disjoint union of at most countable open intervals $I_{j}(e, \theta), 0 \leq j<j(e, \theta)$ (where $j(e, \theta) \in \mathbb{N} \cup\{\infty\}$ ), with $I_{0}(e, \theta)=\left(0, r^{*}(e, \theta)\right.$ ), plus at most a countable set of points. Therefore

$$
\operatorname{meas}(S(e, \theta))=\sum_{0 \leq j<j(e, \theta)} \operatorname{meas}\left(I_{j}(e, \theta)\right) .
$$

Then, there exists ${ }^{3} j_{\delta}<j(e, \theta)$ such that

$$
S_{\delta}(e, \theta):=\bigcup_{0 \leq j \leq j_{\delta}} I_{j}(e, \theta) \subseteq S(e, \theta)
$$

satisfies

$$
\operatorname{meas}\left(S_{\delta}(e, \theta)\right) \geq \operatorname{meas}(S(e, \theta))-\delta .
$$

Consequently we can define a " $\delta$-approximation" of the weak stability boundary setting $\mathcal{W}_{\delta}:=\left\{(r, e, \theta) \in \mathbb{R}^{+} \times[0,1] \times S^{1}\right.$ s.t. $\left.r \in \mathcal{W}_{\delta}(e, \theta)\right\} \quad$ where $\quad \mathcal{W}_{\delta}(e, \theta):=\partial S_{\delta}(e, \theta)$ Remark 2 If $\delta>0$ is the "computational error" the sets $S^{*}(e, \theta)$ in (14) and $S_{\delta}(e, \theta)$ have the same form, being finite union of disjoint intervals ${ }^{4}$. The same holds for $\mathcal{W}, \mathcal{W}(e, \theta)$ in $(15)$ and $\mathcal{W}_{\delta}, \mathcal{W}_{\delta}(e, \theta)$ above.

3 If $j(e, \theta)<\infty$ one can take $j_{\delta}=j(e, \theta)-1$.

4 The fact that the intervals in (14) contain their endpoints is irrelevant since it does not affect the boundary. 


\section{The topology of the WSB}

The topology of the stable set $S(e, \theta)$ is firstly considered (recall (16)), initially showing that $S(e, \theta)$ is bounded.

Lemma 1 If $r_{0}$ is large enough (independently from $e$ and $\left.\theta\right), a\left(r_{0}, e, \theta\right)$-test orbit is never stable, according to Definition 5. In particular, for every $(e, \theta)$ fixed, $S(e, \theta)$ is a bounded subset of $\mathbb{R}^{+}$.

Proof Assume that ${ }^{5} r_{0} \geq 10^{4}$. We claim that $\forall 0 \leq t \leq 4 \pi$

$$
\frac{r_{0}}{2}<r(t)<2 r_{0}, \quad|\Theta(t)|<30 r_{0} .
$$

By the Hamilton's equation for the Hamiltonian $H_{3}$ in (7) we get

$$
\dot{\vartheta}=\frac{\Theta}{r^{2}}-1, \quad|\dot{\Theta}| \leq \frac{2 r}{|r-1|}+r, \quad|\ddot{r}| \leq \frac{\Theta^{2}}{r^{3}}+\frac{1}{r^{2}}+\frac{r+1}{|r-1|}+1 .
$$

Let us prove (19) by contradiction. Assume that there exists $0<T<4 \pi$ such that (19) holds $\forall 0<t<T$ and does not hold for $t=T$. Then we have

$$
|\dot{\Theta}| \leq 3+2 r_{0}, \quad|\ddot{r}| \leq \frac{8 \Theta^{2}}{r_{0}^{3}}+\frac{4}{r_{0}^{2}}+3
$$

$\forall 0<t<T$. Which yields

$$
|\Theta(t)| \leq \Theta_{0}+4 \pi\left(3+2 r_{0}\right) \stackrel{(12)}{\leq} \sqrt{2 r_{0}}+28 r_{0} \leq 29 r_{0},
$$

and

$$
|\ddot{r}| \leq \frac{7200}{r_{0}}+\frac{4}{r_{0}^{2}}+3 \leq 4 \quad \Longrightarrow \quad\left|r(t)-r_{0}\right| \leq 4 \frac{T^{2}}{2} \leq 32 \pi^{2} \leq \frac{r_{0}}{3}
$$

$\forall 0<t<T$. Then we get

$$
|\Theta(T)| \leq 29 r_{0}, \quad\left|r(T)-r_{0}\right| \leq \frac{r_{0}}{3}
$$

namely (19) still holds for $t=T$, which is a contradiction. Recollecting we have proved (19).

By (20) we have that $\forall 0 \leq t \leq 4 \pi$

$$
\dot{\vartheta}(t) \leq \frac{120}{r_{0}}-1 \leq-\frac{1}{2} .
$$

This means that the orbit makes a clockwise turn around the origin in a time $0<T_{*} \leq$ $4 \pi$. In particular, since $r(t) \geq 5000$, it makes a clockwise turn around the Earth and the Moon, and therefore it is not stable (according to Definition 5).

Proof of Proposition 1

Let us fix $(e, \theta) \in(0,1) \times S^{1}$. We first claim that if $\bar{r} \in S(e, \theta)$ the first time $T$ of return on $l$ after making a circle around the Moon is an analytic function of $r$ in an open neighborhood of $\bar{r}$.

5 Obviously we did not make any effort in obtaining the best value of $r_{0}$, since we are interested only in its existence. 
Let us denote by $\psi(t ; \bar{r})=\psi(t ; \bar{r}, e, \theta)$ the flow of the $(\bar{r}, e, \theta)$-test orbit. Let $\vartheta(t ; \bar{r})$ be its $\vartheta$-component. Indeed if $\bar{T}$ is the first time of return w.r.t. $\bar{r}$ then, using the transversality condition

$$
\vartheta(\bar{T} ; \bar{r})=\theta, \quad \partial_{T} \vartheta(\bar{T} ; \bar{r})=\dot{\vartheta}(\bar{T} ; \bar{r})>0
$$

and the implicit function theorem (in analytic $\operatorname{class}^{6}$ ) is applicable to the equation

$$
\vartheta(T ; r)=\theta
$$

Then we find an analytic function $T=T(r)$ such that $\vartheta(T(r) ; r)=\theta$ for every $r$ in an open neighborhood of $\bar{r}$.

Let us define

$$
\begin{gathered}
S_{<0}=S_{<0}(e, \theta):=\left\{r \in S(e, \theta) \text { s.t. } H_{M}(\psi(T ; r))<0\right\} \\
S_{=0}=S_{=0}(e, \theta):=\left\{r \in S(e, \theta) \text { s.t. } H_{M}(\psi(T ; r))=0\right\} .
\end{gathered}
$$

Obviously $S=S_{<0} \cup S_{=0}$.

We first note that $S_{<0}$ is an open subset of $\mathbb{R}^{+}$. If $\bar{r} \in S_{<0}$, for every $r$ in an open neighborhood of $\bar{r}, T(r)$ is well defined, $\dot{\vartheta}(T(r) ; r)>0$ and $H(\psi(T(r) ; r))<0$ by continuity, since the Hamiltonian flow is a diffeomorphism.

The topology of $S_{=0}$ is more involved. Assume that $\bar{r} \in S_{=0}$. Still for every $r \in$ $(\bar{r}-\epsilon, \bar{r}+\epsilon)$ (with $\epsilon$ small enough), $T(r)$ is well defined and $\dot{\vartheta}(T(r) ; r)>0$ by continuity. Let us consider the analytic function

$$
h(r):=H(\psi(T(r) ; r)) .
$$

Take $r \in(\bar{r}-\epsilon, \bar{r}+\epsilon)$; then $r \in S_{=0}$ iff $h(r)=0$. Since $h$ is analytic two cases are possible: $h$ is identically zero on $(\bar{r}-\epsilon, \bar{r}+\epsilon)$ or there are at most a finite number of zeros in $[\bar{r}-\epsilon / 2, \bar{r}+\epsilon / 2]$. Consequently any point of $S_{=0}$ is an inner point or an isolated point. Since the number of isolated points of a subset of $\mathbb{R}$ is at most countable ${ }^{7}, S_{=0}$ is an open set plus, at most, a countable set of points. The same holds for $S$.

Note that, by standard measure theory arguments, any open subset of $\mathbb{R}^{+}$is formed by at most a countable disjoint union of open intervals (with at most one unbounded interval).

We will prove below (see Corollary ${ }^{8} 1$ ) that if $r$ is small enough (uniformly on $e$ and $\theta$ ) then $r \in S_{<0}$. Moreover $S$ is bounded by Lemma 1 . The proof of Proposition 1 is now completed.

Remark 3 It is conceivable that for some $e, \theta$ the set $S(e, \theta)$ does possess isolated points, however to prove it is not straightforward.

6 Note that $\vartheta(T ; r)$ is an analytic function of $T$ and $r$, since the Hamiltonian flow is analytic.

7 Since it can be covered by a union of disjoint open intervals.

8 Actually in Corollary 1 we consider only the case when the eccentricity is larger than $1 / 2$, as the case of eccentricity smaller than $1 / 2$ is simpler (see Remark 4 ). 


\section{The WSB in Delaunay coordinates}

A complete derivation of Delaunay variables can be found in Goldstein et al. (2001), Stiefel and Scheifele (1971) (also see Arnold et al. (2006) and Celletti and Chierchia (2007)). Here we briefly recall the main points.

The osculating ellipse (see Definition 3) is identified by:

- $e=$ eccentricity ,

- $a=$ length of the major semi-axis

- $\gamma=$ argument of the periselenium (measured from the horizontal axis of the inertial frame).

The Delaunay coordinates are symplectic action-angle variables $(L, G, \ell, g)$, where the angles $\ell$ and $g$ are conjugated to the actions $L$ and $G$, respectively, where

- $\ell$ is the mean anomaly measured from the periselenium;

- $g$ is the difference between the argument of the periselenium and the angle describing the position of the Earth (measured from the horizontal axis of the inertial frame);

- $L$ is related to the major semi-axis, $a$, by

$$
L=M^{-1 / 6} \sqrt{a}, \quad \text { where } M:=\text { mass of the Moon; }
$$

- $G$ is the total angular momentum of the spacecraft with respect to the Moon (in the inertial frame), related to the eccentricity and the variable $L$ by

$$
e=\sqrt{1-\frac{G^{2}}{L^{2}}} .
$$

The motions of Earth-Moon-Spacecraft coplanar CR3BP in Delaunay variables are described by the Hamiltonian ${ }^{9}$ (with the standard symplectic form $d L \wedge d \ell+d G \wedge d g$ )

$$
H(L, G, \ell, g):=H^{*}(L, G, r, \rho):=-\frac{1}{2 L^{2}}-G+\epsilon \rho-\epsilon \frac{1}{\sqrt{r^{2}+1-2 \rho}},
$$

9 The actions and the Hamiltonian are firstly scaled as $H_{3}$ in (7):

$$
\tilde{R}:=\mu R, \quad \tilde{\Theta}:=\mu \Theta, \quad \tilde{H}_{3}(\tilde{R}, \tilde{\Theta}, r, \vartheta):=\mu H_{3}(\tilde{R} / \mu, \tilde{\Theta} / \mu, r, \vartheta)
$$

(with symplectic form $d \tilde{R} \wedge d r+d \tilde{\Theta} \wedge d \vartheta$ ) and choosing $\mu:=M^{-2 / 3}$ (see formula (3.3.8) of Celletti and Chierchia (2007)). This yields:

$$
\tilde{H}_{3}=\frac{1}{2 \mu}\left(R^{2}+\frac{\Theta^{2}}{r^{2}}\right)-\frac{\mu M}{r}-\Theta-\frac{\mu(1-M)}{\sqrt{r^{2}+1-2 r \cos (\vartheta+\pi)}}+\mu(1-M) r \cos (\vartheta+\pi) .
$$

Analogously, regarding the two-body Kepler energy $H_{M}$ in (8), it yields:

$$
\tilde{H}_{M}(\tilde{R}, \tilde{\Theta}, r, \vartheta):=\mu H_{M}(\tilde{R} / \mu, \tilde{\Theta} / \mu, r, \vartheta)=\frac{1}{2 \mu}\left(R^{2}+\frac{\Theta^{2}}{r^{2}}\right)-\frac{\mu M}{r} .
$$

Then the Delaunay variables are introduced (see formulas (3.2.21) and (3.2.22) of Celletti and Chierchia (2007)), in which the Hamiltonian $\tilde{H}_{3}$ takes the form $H$ in (26), which is as in formula (3.3.11) of Celletti and Chierchia (2007) (see also (34)). Note that

$$
G=\tilde{\Theta}=\mu \Theta=M^{-2 / 3} \Theta
$$

And again analogously, in Delaunay variables, the two-Body Kepler energy $\tilde{H}_{M}$ takes the form:

$$
\tilde{H}_{M}=-\frac{\mu^{3} M^{2}}{2 L^{2}}=-\frac{1}{2 L^{2}}
$$


where $r$ depends on $L, G, \ell$ and $\rho$ depends on $L, G, \ell, g$ in the following way:

- $r$ is the distance of the spacecraft from the Moon, namely the origin; $r$ is a function of $\ell, L, G$ (actually of $\ell$ and $e=\sqrt{1-G^{2} / L^{2}}$ ); more precisely

$$
r=a(1-e \cos u)=M^{\frac{1}{3}} L^{2}(1-e \cos u)
$$

where $u=u(\ell, e)$ is the eccentric anomaly and is implicitly defined (for $e \in[0,1)$ ) by the Kepler equation

$$
\ell=u-e \sin u
$$

- $\rho:=r \cos (\nu+g)$ where $\nu$ is the true anomaly measured from the periselenium; also $\nu$ is a function of $\ell, L, G$ (actually of $\ell$ and $e=\sqrt{1-G^{2} / L^{2}}$ ) and it is defined by the equation

$$
\tan \frac{\nu}{2}=\sqrt{\frac{1+e}{1-e}} \tan \frac{u}{2}
$$

$\epsilon$ is a scaled mass:

$$
\epsilon:=M^{\frac{-2}{3}}(1-M)
$$

Note that

$$
\begin{aligned}
\rho=r \cos (\nu+g) & =a\left(\cos g \cos u-e \cos g-\frac{G}{L} \sin g \sin u\right) \\
& =L^{2} M^{\frac{1}{3}}\left(\cos g \cos u-e \cos g-\sqrt{1-e^{2}} \sin g \sin u\right) .
\end{aligned}
$$

Recalling (25) the two-Body Kepler energy in Delaunay variables has the form

$$
H_{M}=-\frac{M^{2 / 3}}{2 L^{2}} .
$$

This two-Body Kepler energy is not the one corresponding to our Hamiltonian (26) but the one directly obtained applying the changes of coordinates to (8) (i.e. without scaling with $\mu$ ). However, as we are only interested in the sign of this function, the two are equivalent, since we can pass from one to the other by scaling with a positive factor.

Furthermore, note that $H_{M}<0$. Indeed, by construction, Delaunay variables describe only motions with $H_{M}<0$. This is not a problem here. As a matter of fact in the following we are interested in showing (analytically) that orbits starting sufficiently close to the Moon are stable according to Definition 5.

By (27) we also have

$$
H_{M}=\frac{M(e \cos u-1)}{2 r},
$$

which at the periselenium, namely when $\nu=u=\ell=0$, is

$$
H_{M}=\frac{M(e-1)}{2 r},
$$

(see formula (3.2.22) of Celletti and Chierchia (2007)).

However if we directly apply this changes of coordinates to (8)(i.e. without scaling by the positive factor $\mu$ ), the two-Body Kepler energy in Delaunay variables takes the form:

$$
H_{M}=-\frac{1}{2 \mu L^{2}}=-\frac{M^{2 / 3}}{2 L^{2}} .
$$


which is the expression usually used by Belbruno.

In the rotating frame $\mathbf{F 2}$ the angle $\vartheta$ as function of the Delaunay variables is

$$
\vartheta=\nu+g-\pi
$$

since $\nu+g$ is the angle between the Moon-Spacecraft line and the Moon-Earth line (see Figure (2)). In the rotating frame $\mathbf{F} 2$ we say that an orbit $(r(t), \vartheta(t))$ of the Spacecraft starting at time $t=t_{0}$ makes an (anti-clockwise) circle around the Moon in a time $T>0$ if

$$
\int_{t_{0}}^{t_{0}+T} \frac{d}{d t} \vartheta d t=\int_{t_{0}}^{t_{0}+T} \frac{d}{d t}(\nu+g) d t=2 \pi
$$

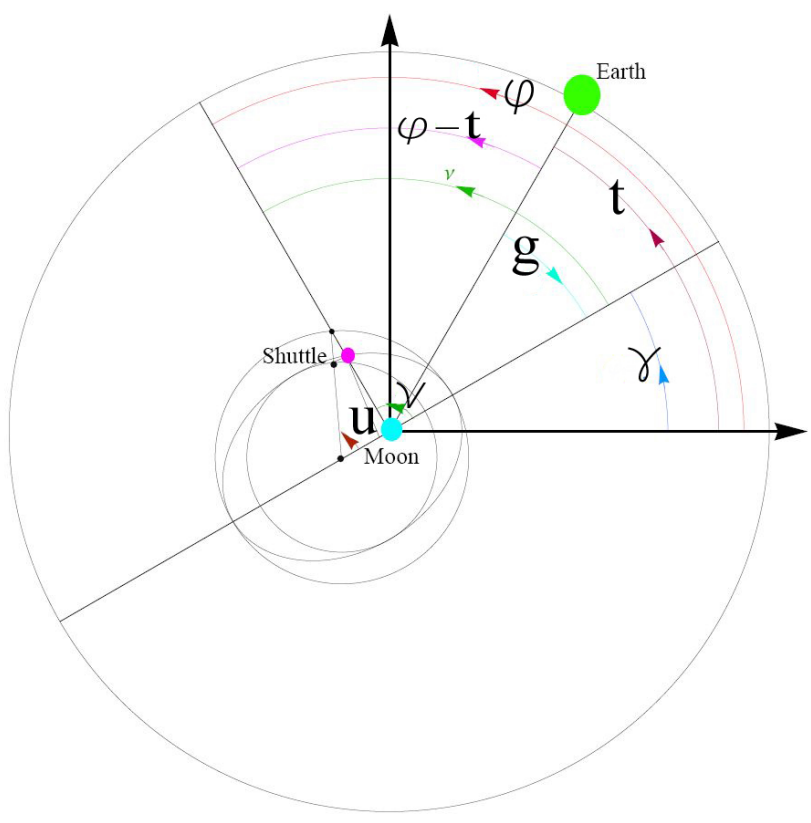

Fig. 2 The angle variables describing the system

A $\left(r_{0}, e_{0}, \vartheta_{0}\right)$-test orbit (see Definition 4$)$ in Delaunay variables has:

1) $\nu(t)=0$ or $\pi$ (periselenium or aposelenium) $\stackrel{(29)}{\Longrightarrow} u(t)=0$ or $\pi \stackrel{(28)}{\Longrightarrow} \ell(0)=0$ or $\pi$

2) $\vartheta(0)=\vartheta_{0}$, (starting on the line $l$ with inclination $\left.\vartheta_{0}\right) \stackrel{(34)}{\Longrightarrow} g(0)=\vartheta_{0}+\pi$ or $\vartheta_{0}$

3) $r(0)=r_{0}$ (starting at a distance $r_{0}$ from the Moon $) \stackrel{(27)}{\Longrightarrow} L(0)=\sqrt{r_{0} M^{-1 / 3} /\left(1 \mp e_{0}\right)}$

4) $e(0)=e_{0}$ (starting with eccentricity $\left.e_{0}\right) \stackrel{(21)}{\Longrightarrow} G(0)= \pm L(0) \sqrt{1-e_{0}^{2}}(+/-$ for direct/retrograde direction). 
Recollecting a $\left(r_{0}, e_{0}, \vartheta_{0}\right)$-test orbit has initial data

$L(0)=\sqrt{r_{0} M^{-1 / 3 /\left(1 \mp e_{0}\right)}}(-/+$ for periselenium/aposelenium $)$,

$G(0)= \pm \sqrt{r_{0} M^{-1 / 3}\left(1 \pm e_{0}\right)} \quad(+/-$ for direct/retrograde and periselenium/aposelenium $)$,

$\ell(0)=0$ or $\pi$ (periselenium or aposelenium),

$\vartheta(0)=\vartheta_{0}$

(recall also (12) and (24)).

The equation of motions of the Hamiltonian $H$ in (26) are:

$$
\begin{aligned}
\dot{\ell}= & \partial_{L} H=\frac{d H^{*}}{d L} \\
= & \partial_{L} H^{*}+\partial_{r} H^{*} \partial_{L} r+\partial_{r} H^{*} \partial_{e} r \partial_{L} e+\partial_{r} H^{*} \partial_{u} r \partial_{e} u \partial_{L} e \\
& +\partial_{\rho} H^{*} \partial_{L} \rho+\partial_{\rho} H^{*} \partial_{e} \rho \partial_{L} e+\partial_{\rho} H^{*} \partial_{u} \rho \partial_{e} u \partial_{L} e \\
\dot{g}= & \partial_{G} H=\frac{d H^{*}}{d G} \\
= & \partial_{G} H^{*}+\partial_{r} H^{*} \partial_{e} r \partial_{G} e+\partial_{r} H^{*} \partial_{u} r \partial_{e} u \partial_{G} e+\partial_{\rho} H^{*} \partial_{e} \rho \partial_{G} e+\partial_{\rho} H^{*} \partial_{u} \rho \partial_{e} u \partial_{G} e \\
\dot{L}= & -\partial_{\ell} H=-\frac{d H^{*}}{d \ell}=-\partial_{r} H^{*} \partial_{u} r \partial_{\ell} u-\partial_{\rho} H^{*} \partial_{u} \rho \partial_{\ell} u \\
\dot{G}= & -\partial_{g} H=-\frac{d H^{*}}{d g}=-\partial_{\rho} H^{*} \partial_{g} \rho .
\end{aligned}
$$

where

$$
\begin{aligned}
& \partial_{L} H^{*}=\frac{1}{L^{3}}, \quad \partial_{G} H^{*}=-1, \quad \partial_{r} H^{*}=\frac{\epsilon r}{\left(\sqrt{r^{2}+1-2 \rho}\right)^{3}}, \\
& \partial_{\rho} H^{*}=\epsilon\left(1-\frac{1}{\left(\sqrt{r^{2}+1-2 \rho}\right)^{3}}\right),
\end{aligned}
$$

with

$$
r=r(L, e, u) \stackrel{(27)}{=} M^{\frac{1}{3}} L^{2}(1-e \cos u)
$$

and

$$
\rho=\rho(L, g, e, u) \stackrel{(30)}{=} L^{2} M^{\frac{1}{3}}\left(\cos g \cos u-e \cos g-\sqrt{1-e^{2}} \sin g \sin u\right) .
$$

therefore

$$
\begin{aligned}
& \partial_{L} r=2 L M^{\frac{1}{3}}(1-e \cos u) \\
& \partial_{u} r=e L^{2} M^{\frac{1}{3}} \sin u \\
& \partial_{e} r=-L^{2} M^{\frac{1}{3}} \cos u \\
& \partial_{u} \rho=-L^{2} M^{\frac{1}{3}}\left(\sqrt{1-e^{2}} \sin g \cos u+\cos g \sin u\right) \\
& \partial_{e} \rho=L^{2} M^{\frac{1}{3}}\left(-\cos g+\frac{e}{\sqrt{1-e^{2}}} \sin g \sin u\right) \\
& \partial_{g} \rho=L^{2} M^{\frac{1}{3}}\left(e \sin g-\cos u \sin g-\sqrt{1-e^{2}} \cos g \sin u\right) \\
& \partial_{L} \rho=2 L M^{\frac{1}{3}}\left(\cos g \cos u-e \cos g-\sqrt{1-e^{2}} \sin g \sin u\right)
\end{aligned}
$$


Moreover, since $e=\sqrt{1-\frac{G^{2}}{L^{2}}}$ and $u=u(\ell, e)$ solves the Kepler equation $\ell=u-e \sin u$, we get

$$
\begin{aligned}
& \partial_{L} e=\frac{G^{2}}{L^{3} \sqrt{1-\frac{G^{2}}{L^{2}}}}=\frac{\left(1-e^{2}\right)}{e L} \\
& \partial_{G} e=-\frac{G}{L^{2} \sqrt{1-\frac{G^{2}}{L^{2}}}}=-\frac{\sqrt{1-e^{2}}}{e L} \\
& \partial_{e} u=\sin u+e \cos u \partial_{e} u \Rightarrow \partial_{e} u=\frac{\sin u}{1-e \cos u} \\
& \partial_{\ell} u=1+e \cos u \partial_{\ell} u \quad \Rightarrow \partial_{\ell} u=\frac{1}{1-e \cos u} .
\end{aligned}
$$

In conclusion

$$
\begin{aligned}
& \dot{\ell}= \partial_{L} H= \\
& \frac{1}{L^{3}}+ \epsilon M^{\frac{1}{3}} L\left(1-\frac{1}{\left(\sqrt{1-2 r \cos (v+g)+r^{2}}\right)^{3}}\right)\left[\cos g\left(2 \cos u-e-\frac{1}{e}-\frac{\sin ^{2} u\left(1-e^{2}\right)}{e(1-e \cos u)}\right)\right. \\
&+\sin g\left(-\sqrt{1-e^{2}} \sin u\left(1+\frac{\left(1-e^{2}\right) \cos u}{e(1-e \cos u)}\right)\right]+\epsilon M^{\frac{2}{3}} L^{3}\left(\frac{1}{\left(\sqrt{1-2 r \cos (v+g)+r^{2}}\right)^{3}}\right) . \\
& \cdot\left[3-3 e \cos u+2 e^{2} \cos ^{2} u-\frac{1}{e} \cos u-e^{2}\right] \\
& \dot{g}= \partial_{G} H= \\
&-1+ \epsilon M^{\frac{1}{3}} L\left(1-\frac{1}{\left(\sqrt{1-2 r \cos (v+g)+r^{2}}\right)^{3}}\right)\left[\cos g\left(\frac{\sqrt{1-e^{2}}}{e}\left(1+\frac{\sin ^{2} u}{(1-e \cos u)}\right)\right)+\right. \\
&\left.\quad+\sin g \sin u\left(\frac{-e+\cos u}{e(1-e \cos u)}\right)\right]+\frac{\epsilon M^{\frac{2}{3}} L^{3}}{\left(\sqrt{1-2 r \cos (v+g)+r^{2}}\right)^{3}}\left[\frac{\sqrt{1-e^{2}}}{e}(\cos u-e)\right] \\
& \dot{L}=-\partial_{\ell} H= \\
&-\epsilon M^{\frac{1}{3}} L^{2}\left(-1+\frac{1}{\left(\sqrt{1-2 r \cos (v+g)+r^{2}}\right)^{3}}\right)\left[\cos g\left(\frac{\sin u}{1-e \cos u}\right)+\sin g\left(\frac{\sqrt{1-e^{2}} \cos u}{1-e \cos u}\right)\right] \\
& \quad-\frac{\epsilon M^{\frac{2}{3}} L^{4}}{\left(\sqrt{1-2 r \cos (v+g)+r^{2}}\right)^{3}}(e \sin u) .
\end{aligned}
$$

$$
\begin{aligned}
& \dot{G}=-\partial_{g} H= \\
& -\epsilon M^{\frac{1}{3}} L^{2}\left(-1+\frac{1}{\left(\sqrt{1-2 r \cos (v+g)+r^{2}}\right)^{3}}\right)\left(\sin g(\cos u-e)+\sqrt{1-e^{2}} \cos g \sin u\right)
\end{aligned}
$$

Since $G$ appears in the Hamiltonian vector field only through $e$, it is convenient to use $e$ instead of $G$ as independent variable. Then the value of $G$ is recovered by the formula

$$
G=L \sqrt{1-e^{2}}
$$

The equation for $\dot{e}$ is:

$$
\begin{aligned}
& \dot{e}=\partial_{L} e \dot{L}+\partial_{G} e \dot{G}= \\
& -\epsilon M^{\frac{1}{3}} L\left(-1+\frac{1}{\left(\sqrt{1-2 r \cos (v+g)+r^{2}}\right)^{3}}\right)\left[\cos g\left(\frac{\left(1-e^{2}\right) \sin u \cos u}{1-e \cos u}\right)+\sin g \frac{\sqrt{1-e^{2}}}{1-e \cos u}\right. \\
& \left.\quad\left(-2 e \cos u+1+\cos ^{2} u\right)\right]-\frac{\epsilon M^{\frac{2}{3}} L^{3}}{\left(\sqrt{1-2 r \cos (v+g)+r^{2}}\right)^{3}}\left(\left(1-e^{2}\right) \sin u\right)=: w(L, e, \ell, g) .
\end{aligned}
$$


To sum up, in the variables $(L, e, \ell, g)$, the equation of motions are

$$
\dot{L}=-\partial_{\ell} H, \quad \dot{e}=w, \quad \dot{\ell}=\partial_{L} H, \quad \dot{g}=\partial_{G} H .
$$

\section{Analytic estimates on the stable zone around the Moon}

The aim of this section is to obtain a realistic, analytic estimate from below on $r^{*}$ defined in Proposition 1.

From the equations of motion it will be deduced that, if the Spacecraft starts close enough to the Moon, then the Mean Anomaly is a so called "fast angle". This means that the Spacecraft actually comes back to the starting line $l$ in a time of first return $T$ which is quite short, allowing to estimate the variation of all the other variables over one revolution.

For some fixed

$$
0<L_{\max }, \quad 1 / 2 \leq e_{\min }<e_{\max }<1
$$

satisfying

$$
r_{\max }:=M^{1 / 3} L_{\max }^{2}\left(1+e_{\max }\right)<1,
$$

we set

$$
\Omega:=\left\{(L, e, \ell, g) \mid L \in\left(0 ; L_{\max }\right), \quad e \in\left(e_{\min } ; e_{\max }\right), \ell, g \in \mathbb{T}^{1}\right\} .
$$

Notice that, by (27), in $\Omega$

$$
0<r<r_{\max }<1 .
$$

Remark 4 In view of applications (i.e. the comparison with Hiten's mission data) we are interested to values of $e$ close to 1 , so we assume

$$
e_{\min } \geq 1 / 2 \text {. }
$$

However it is possible to prove that better estimates can be obtained for smaller values of the eccentricity.

Let us define

$$
S_{0}:=\inf _{\Omega} \partial_{L} H, \quad S_{1}:=-\inf _{\Omega} \partial_{G} H, \quad S_{2}:=\sup _{\Omega}\left|\partial_{\ell} H\right|, \quad S_{3}:=\sup _{\Omega}|w| .
$$

The next Lemma, whose proof can be found in Appendix 6, contains some analytic estimates on $S_{0}, S_{1}, S_{2}, S_{3}$ in terms of $L_{\max }, e_{\min }, e_{\max }$ (defined in (46)).

Lemma 2 We have that

$$
S_{0} \geq \tilde{S}_{0}, \quad \sup _{\Omega}\left|1+\partial_{G} H\right| \leq \tilde{S}_{1} \Longrightarrow S_{1} \leq 1+\tilde{S}_{1}, \quad S_{2} \leq \tilde{S}_{2}, \quad S_{3} \leq \tilde{S}_{3},
$$

where

$$
\begin{aligned}
\tilde{S}_{0}:= & \frac{1}{L_{\max }^{3}}-\epsilon M^{\frac{1}{3}} L_{\max }\left(\frac{1}{\left(1-r_{\max }\right)^{3}}-1\right) \frac{\left(1+e_{\min }\right)^{2}}{e_{\min }} \\
& -\epsilon M^{\frac{2}{3}} L_{\max }^{3}\left(\frac{1}{\left(1-r_{\max }\right)^{3}}\right) \frac{\left(1+e_{\max }\right)^{3}}{e_{\max }}
\end{aligned}
$$




$$
\begin{aligned}
& \tilde{S}_{1}:= \epsilon M^{\frac{1}{3}} L_{\max }\left(\frac{1}{\left(1-r_{\max }\right)^{3}}-1\right) \frac{\sqrt{3+2 e_{\min }-e_{\min }^{2}}}{e_{\min }} \\
&+\frac{\epsilon M^{\frac{2}{3}} L_{\max }^{3}}{\left(1-r_{\max }\right)^{3}} \frac{\sqrt{1-e_{\min }^{2}}}{e_{\min }}\left(1+e_{\min }\right) \\
& \tilde{S}_{2}:=\epsilon M^{\frac{1}{3}} L_{\max }^{2}\left(\frac{1}{\left(1-r_{\max }\right)^{3}}-1\right) \sqrt{\frac{1+e_{\max }}{1-e_{\max }}}+\frac{\epsilon M^{\frac{2}{3}} L_{\max }^{4}}{\left(1-r_{\max }\right)^{3}} e_{\max } \\
& \tilde{S}_{3}:=\epsilon M^{\frac{1}{3}} L_{\max }\left(\frac{1}{\left(1-r_{\max }\right)^{3}}-1\right) 2 \sqrt{1-e_{\min }^{2}}+\frac{\epsilon M^{\frac{2}{3}} L_{\max }^{3}}{\left(1-r_{\max }\right)^{3}}\left(1-e_{\min }^{2}\right) .
\end{aligned}
$$

Note that

$$
\tilde{S}_{0} \rightarrow \infty, \quad \tilde{S}_{1}, \tilde{S}_{2} / L_{\max }, \tilde{S}_{3} \rightarrow 0, \quad \text { as } L_{\max } \rightarrow 0
$$

In the following we will assume that

$$
\begin{aligned}
& \inf _{\Omega} \partial_{L} H=S_{0}>0, \quad-S_{1}=\inf _{\Omega} \partial_{G} H \leq \sup _{\Omega} \partial_{G} H \leq 0, \quad S_{0} \geq 5 S_{1}, \\
& S_{2} \tilde{T}<L_{\max }, \quad 2 S_{3} \tilde{T}<e_{\max }-e_{\min } \quad \text { where } \tilde{T}=2 \pi /\left(S_{0}-S_{1}\right) .
\end{aligned}
$$

Note that from (58) these conditions can always be assumed if $L_{\max }$ is small enough.

Then we can choose

$$
\begin{aligned}
& 0<\tilde{L}_{\max }:=L_{\max }-S_{2} \tilde{T}<L_{\max }, \\
& e_{\min }<\tilde{e}_{\min }:=e_{\min }+S_{3} \tilde{T}<\tilde{e}_{\max }:=e_{\max }-S_{3} \tilde{T}<e_{\max }
\end{aligned}
$$

and set

$$
\tilde{\Omega}:=\left\{(L, e, \ell, g) \mid L \in\left(0, \tilde{L}_{\max }\right], \quad e \in\left[\tilde{e}_{\min }, \tilde{e}_{\max }\right], \ell, g \in \mathbb{T}^{1}\right\}
$$

Lemma 3 Assuming (59) and (60). Then

$$
(L(0), e(0), \ell(0), g(0)) \in \tilde{\Omega} \quad \Longrightarrow \quad(L(t), e(t), \ell(t), g(t)) \in \Omega, \quad \forall t \in[0, \tilde{T}] .
$$

Proof Assume by contradiction that $\exists T_{*} \in(0, \tilde{T})$ such that $(L(t), e(t), \ell(t), g(t)) \in \Omega$ for every $t \in\left[0, T_{*}\right)$, but $\left(L\left(T_{*}\right), e\left(T_{*}\right), \ell\left(T_{*}\right), g\left(T_{*}\right)\right) \notin \Omega$. Then (at least) one of the following equalities occurs:

$$
L\left(T_{*}\right)=L_{\max }, \quad e\left(T_{*}\right)=e_{\min }, \quad e\left(T_{*}\right)=e_{\max } .
$$

But this is in contradiction with (61) from (45), (59) and (60), proving the Lemma. 
Proposition 2 Assume (59) and (60). A ( $\left.r_{0}, e_{0}, \theta_{0}\right)$-test orbit with

$$
0<r_{0}<r^{*}:=M^{1 / 3} \tilde{L}_{\max }^{2}\left(1-\tilde{e}_{\max }\right), \quad e_{0} \in\left(\tilde{e}_{\min }, \tilde{e}_{\max }\right)
$$

is stable according to Definition 5. In particular:

i) $r(t)<1$ for every $t \in[0, \tilde{T}]$, namely it cannot go around the Earth or crash into the Earth up to time $\tilde{T}$

ii) there exists $0<T \leq \tilde{T}$ such that

$$
\int_{0}^{T} \dot{\nu}+\dot{g}=2 \pi
$$

namely the orbit makes a circle around the Moon in time T.

Moreover

$$
\dot{\vartheta}(T)=\dot{\nu}(T)+\dot{g}(T)>0
$$

Remark 5 In the proposition above we consider the worst case, which is when the test orbit starts at periselenium (see the minus sign in equation (63) and recall (36)). Better estimates hold for the aposelenium case (in particular replace $1-\tilde{e}_{\max }$ with $1+\tilde{e}_{\min }$ in formula (63)).

Proof. Let us first note that, by (27) and (63) the test orbits starts in $\tilde{\Omega}$. Then, by Lemma 3 , it remains in $\Omega$ for every $t \in[0, \tilde{T}]$. In particular, by $(49), r(t)<1$ for every $t \in[0, \tilde{T}]$.

Let us consider only the case $\ell(0)=0$, the case $\ell(0)=\pi$ being analogous We claim that there exists $0<T_{*} \leq \bar{T}$ such that

$$
\int_{0}^{T_{*}} \dot{\ell}+\dot{g}=2 \pi \quad \text { and } \quad 2 \pi \leq \int_{0}^{T_{*}} \dot{\ell} \leq 3 \pi .
$$

Since $\dot{\ell}+\dot{g} \geq S_{0}-S_{1}>0$ the existence of (a unique) $0<T_{*} \leq \bar{T}$ satisfying the equality in (65) follows. Also the first inequality in (65) is trivial since $\dot{g} \leq 0$. It remains to prove that $\int_{0}^{T_{*}} \dot{\ell} \leq 3 \pi$. Assume by contradiction that $\int_{0}^{T_{*}} \dot{\ell}>3 \pi$. Then

$$
2 \pi=\int_{0}^{T_{*}} \dot{\ell}+\dot{g}>3 \pi-S_{1} T_{*} \geq 3 \pi-S_{1} \bar{T} \geq 2 \pi
$$

since $S_{0} \geq 3 S_{1}$. This proves $(65)$.

Note that the solution $u=u(\ell)$ of the Kepler equation (28) and $\nu=\nu(u)$ in (29) satisfy $u(n \pi)=n \pi=\nu(n \pi)$

$$
\nu(u) \geq u, \quad u(\ell) \geq \ell \quad \text { when } \quad u, \ell \in[0, \pi]+2 n \mathbb{Z} .
$$

In particular $\nu(u(\ell)) \geq \ell$ for $\ell \in[0, \pi]+2 n \mathbb{Z}$. Since by $(65) \ell\left(T_{*}\right) \in[2 \pi, 3 \pi]$ we get $\nu\left(T_{*}\right)=\nu\left(u\left(\ell\left(T_{*}\right)\right)\right) \geq \ell\left(T_{*}\right)$. Then, since $\nu(0)=\nu(u(\ell(0)))=\nu(u(0))=0$,

$$
\int_{0}^{T_{*}} \dot{\nu}+\dot{g}=\nu\left(T_{*}\right)+\int_{0}^{T_{*}} \dot{g} \geq \ell\left(T_{*}\right)+\int_{0}^{T_{*}} \dot{g}=\int_{0}^{T_{*}} \dot{\ell}+\dot{g}=2 \pi .
$$

The existence of $T$ in (64) follows by continuity. 
Using (28) and (29) and denoting $u^{t}:=u(\ell(t)), \nu^{t}:=\nu\left(u^{t}\right)$ we compute for $0 \leq$ $t \leq T$

$$
\begin{aligned}
\dot{\nu}(t) & =\frac{d \nu}{d u}\left(u^{t}\right) \frac{d u}{d \ell}(l(t)) \dot{\ell}(t) \\
& =\left(1+\frac{1+e}{1-e} \tan ^{2}\left(u^{t} / 2\right)\right)^{-1} \sqrt{\frac{1+e}{1-e}} \frac{1}{\cos ^{2}\left(u^{t} / 2\right)} \frac{1}{1-e \cos u^{t}} \dot{\ell}(t) \\
& =\left(1+\tan ^{2}\left(\nu^{t} / 2\right)\right)^{-1} \sqrt{\frac{1+e}{1-e}} \frac{1}{\cos ^{2}\left(u^{t} / 2\right)} \frac{1}{1-e \cos u^{t}} \dot{\ell}(t)>0 .
\end{aligned}
$$

Denoting $\nu_{*}:=\nu(T)-2 \pi$ we have that $0 \leq \nu_{*} \leq \pi / 2$. Indeed $0 \leq \nu_{*}$ follows since $\dot{\nu}>0$. On the other hand (64) implies that

$$
\nu_{*}=-\int_{0}^{T} \dot{g} \leq S_{1} T \leq 2 \pi \frac{S_{1}}{S_{0}-S_{1}} \stackrel{S_{0} \geq 5 S_{1}}{\leq} \frac{\pi}{2} .
$$

Noting that $u_{*}:=u(T)-2 \pi$ satisfies $0 \leq u_{*} \leq \nu_{*} \leq \pi / 2$ (recall (66)), by (67) we get

$$
\dot{\nu}(T)>\left(1+\tan ^{2}\left(\nu_{*} / 2\right)\right)^{-1} \dot{\ell}(T) \geq \dot{\ell}(T) / 2 \geq S_{0} / 2 .
$$

Then

$$
\dot{\nu}(T)+\dot{g}(T) \geq S_{0} / 2-S_{1} \geq 3 S_{1} / 2>0 .
$$

The proposition is proved.

\section{Corollary 1 Choose}

$$
0<\tilde{L}_{\max }<L_{\max }, \quad \frac{1}{2} \leq e_{\min }<\tilde{e}_{\min }<\tilde{e}_{\max }<e_{\max } \leq 1
$$

satisfying (47), (59), (60) and (61).

Then

$$
S(e, \theta) \supset\left(0, r^{*}\right) \quad \forall e \in\left(\tilde{e}_{\min }, \tilde{e}_{\max }\right) \quad \forall \theta \in S^{1},
$$

where $r^{*}$ was defined in (63).

\subsection{An example}

We now explicitly evaluate the value of $r^{*}(e)$ when $e=0.95$, which is the initial eccentricity used by Belbruno in the Hiten mission (see p.145 of Belbruno (2004)).

We have to choose parameters satisfying the hypotheses of Corollary 1 . We proceed in two ways. The former is analytic and uses Lemma 2 to estimate $S_{i}, i=0,1,2,3$ in (52). The latter is numerical and uses Mathematica to estimates the $\sup _{\Omega}$ and $\inf _{\Omega}$ in (52).

In the first case we note that the conditions

$$
\begin{aligned}
& \tilde{S}_{0}>0, \quad \tilde{S}_{1}<1, \quad \tilde{S}_{0} \geq 5\left(\tilde{S}_{1}+1\right) \\
& \tilde{S}_{2} \bar{T}<L_{\max }, \quad 2 \tilde{S}_{3} \bar{T}<e_{\max }-e_{\min } \quad \text { with } \bar{T}:=2 \pi /\left(\tilde{S}_{0}-\tilde{S}_{1}-1\right)
\end{aligned}
$$

imply (59)-(60), from (52) and $\bar{T} \geq \tilde{T}$. 
Taking

$$
L_{\max }:=0.271337, \quad e_{\min }:=0.938443, \quad e_{\max }:=0.961557
$$

which implies, by (47):

$$
r_{\max }:=0.0333365
$$

we get

$\tilde{S}_{0}=49.3889, \quad \tilde{S}_{1}=0.280378, \quad \tilde{S}_{2}=0.246627, \quad \tilde{S}_{3}=0.0884847, \quad \bar{T}=0.130604$.

Then (47) and (68) are satisfied. By (61)

$$
\tilde{L}_{\max }=0.239126, \quad \tilde{e}_{\min }=0.4999837, \quad \tilde{e}_{\max }=0.950000057
$$

and $0.95 \in\left[\tilde{e}_{\min }, \tilde{e}_{\max }\right]$. By $(63)$ we get

$$
r^{*}(0.95)=0.000659972
$$

Since the escaping radius used by Belbruno in the Hiten mission was $r_{B e l}:=0.00478148$ we get

$$
r_{B e l} / r^{*}(0.95)=7.24497
$$

The value of $r^{*}$ can be slightly improved numerically estimating $S_{0}, S_{1}, S_{2}, S_{3}$ in (51), e.g. by Mathematica. Taking

$$
L_{\max }:=0.3735, \quad e_{\min }:=0.899, \quad e_{\max }:=0.9999
$$

we get

$S_{0}=18.4422, \quad S_{1}=1.11143, \quad S_{2}=0.0367016, \quad S_{3}=0.137461, \quad \tilde{T}=0.362545$.

Then (47), (59) and (60) are satisfied. By (61)

$$
\tilde{L}_{\max }=0.360194, \quad \tilde{e}_{\min }=0.948836, \quad \tilde{e}_{\max }=0.950064,
$$

and $0.95 \in\left[\tilde{e}_{\min }, \tilde{e}_{\max }\right]$. By $(63)$ we get

$$
r^{*}(0.95)=0.0014955
$$

In this case we have that

$$
r_{\text {Bel }} / r^{*}(0.95)=3.19725 \text {. }
$$




\section{Appendix}

We prove Lemma 2. We have

$$
\begin{aligned}
& \min _{\Omega} \partial_{L} H \geq \frac{1}{L_{\max }^{3}}-\epsilon M^{\frac{1}{3}} L_{\max } \max _{\Omega}\left\{\left|1-\frac{1}{\left(\sqrt{1-2 r \cos (v+g)+r^{2}}\right)^{3}}\right|\right\} \\
& \max _{\Omega}\left\{\mid \cos g\left(2 \cos u-e-\frac{1}{e}-\frac{\sin ^{2} u\left(1-e^{2}\right)}{e(1-e \cos u)}\right)\right. \\
& \left.+\sin g\left(-\sqrt{1-e^{2}} \sin u\left(1+\frac{\left(1-e^{2}\right) \cos u}{e(1-e \cos u)}\right)\right) \mid\right\} \\
& -\epsilon M^{\frac{2}{3}} L_{\max }^{3} \frac{1}{\left(1-r_{\max }\right)^{3}} \max _{\Omega}\left\{\left|3-3 e \cos u+2 e^{2} \cos ^{2} u-\frac{1}{e} \cos u-e^{2}\right|\right\} \\
& \geq \frac{1}{L_{\max }^{3}}-\epsilon M^{\frac{1}{3}} L_{\max }\left(\frac{1}{\left(1-r_{\max }\right)^{3}}-1\right) \underset{e \in\left[e_{\min }, e_{\max }\right], u \in[0,2 \pi]}{\max }\left\{\left(\frac{1}{4 e^{2}(1-e \cos u)}\right.\right. \\
& \left(10+22 e^{2}+8 e^{4}-3 e\left(7+13 e^{2}\right) \cos u+6\left(-1+3 e^{2}+2 e^{4}\right) .\right. \\
& \left.\left.\left.\cdot \cos (2 u)+\left(e-e^{3}-4 e^{5}\right) \cos (3 u)\right)\right)^{\frac{1}{2}}\right\}-\epsilon M^{\frac{2}{3}} L_{\max }^{3}\left(\frac{1}{\left(1-r_{\max }\right)^{3}}\right) \\
& \max _{e \in\left[e_{\text {min }}, e_{\text {max }}\right], u \in[0,2 \pi]}\left\{\left|3-3 e \cos u+2 e^{2} \cos ^{2} u-\frac{1}{e} \cos u-e^{2}\right|\right\} \\
& \geq \frac{1}{L_{\max }^{3}}-\epsilon M^{\frac{1}{3}} L_{\max }\left(\frac{1}{\left(1-r_{\max }\right)^{3}}-1\right) \max _{u \in[0,2 \pi]}\left\{\mid\left(\frac{1}{4 e_{\min }^{2}\left(1-e_{\min } \cos u\right)}\right.\right. \\
& \left(10+22 e_{m i n}^{2}+8 e_{m i n}^{4}-3 e_{m i n}\left(7+13 e_{m i n}^{2}\right) \cos u+6\left(-1+3 e_{m i n}^{2}+2 e_{m i n}^{4}\right) .\right. \\
& \left.\left.\left.\cdot \cos (2 u)+\left(e_{\min }-e_{\min }^{3}-4 e_{\min }^{5}\right) \cos (3 u)\right)\right)^{\frac{1}{2}} \mid\right\}-\epsilon M^{\frac{2}{3}} L_{\max }^{3}\left(\frac{1}{\left(1-r_{\max }\right)^{3}}\right) \\
& \max _{e \in\left[e_{\min }, e_{\max }\right]}\left\{\frac{(1+e)^{3}}{e}\right\}
\end{aligned}
$$

where it has been used that

$$
\max _{g \in[0,2 \pi]}(A \cos g+B \sin g)=\sqrt{A^{2}+B^{2}}
$$

and it has been noted that, by (50), the function

$$
\begin{aligned}
& \left(\frac { 1 } { 4 e ^ { 2 } ( 1 - e \operatorname { c o s } u ) } \left(10+22 e^{2}+8 e^{4}-3 e\left(7+13 e^{2}\right) \cos u+6\left(-1+3 e^{2}+2 e^{4}\right) \cos (2 u)+\right.\right. \\
& \left.\left.+\left(e-e^{3}-4 e^{5}\right) \cos (3 u)\right)\right)^{\frac{1}{2}}
\end{aligned}
$$

reaches its maximum in $u=\pi$ leading to the decreasing function $(1+e)^{2} / e$.

The function

$$
\left|3-3 e \cos u+2 e^{2} \cos ^{2} u-\frac{1}{e} \cos u-e^{2}\right|
$$


has two relative maxima in $u=0$ and $u=\pi \forall e \in(0,1], u \in[0,2 \pi]$; therefore the absolute maximum is reached for $u=\pi$. Finally $(1+e)^{3} / e$ is increasing by (50). This proves (53).

Analogously:

$$
\begin{aligned}
& \sup _{\Omega}\left|1+\partial_{G} H\right| \\
& \leq \epsilon M^{\frac{1}{3}} L_{\max } \max _{\Omega}\left|1-\frac{1}{\left(\sqrt{1-2 r \cos (v+g)+r^{2}}\right)^{3}}\right| \\
& \cdot \max _{\Omega}\left|\cos g\left(\frac{\sqrt{1-e^{2}}}{e}\left(1+\frac{\sin ^{2} u}{(1-e \cos u)}\right)\right)+\sin g \sin u\left(\frac{-e+\cos u}{e(1-e \cos u)}\right)\right| \\
& +\frac{\epsilon M^{\frac{2}{3}} L_{\max }^{3}}{\left(1-r_{\max }\right)^{3}} \max _{\Omega}\left|\frac{\sqrt{1-e^{2}}}{e}(\cos u-e)\right| \\
& \stackrel{(72)}{\leq} \epsilon M^{\frac{1}{3}} L_{\max }\left(\frac{1}{\left(1-r_{\max }\right)^{3}}-1\right)_{e \in\left[e_{\min }, e_{\max }\right], u \in[0,2 \pi]} \sqrt{f_{1}(e, \cos u)} \\
& +\frac{\epsilon M^{\frac{2}{3}} L_{\max }^{3}}{\left(1-r_{\max }\right)^{3}} \max _{e \in\left[e_{\min }, e_{\max }\right]} \frac{\sqrt{1-e^{2}}}{e}(1+e) \\
& \leq \epsilon M^{\frac{1}{3}} L_{\max }\left(\frac{1}{\left(1-r_{\max }\right)^{3}}-1\right) \max _{u \in[0,2 \pi]} \sqrt{f_{1}\left(e_{\min }, \cos u\right)} \\
& +\frac{\epsilon M^{\frac{2}{3}} L_{\max }^{3}}{\left(1-r_{\max }\right)^{3}} \frac{\sqrt{1-e_{\min }^{2}}}{e_{\min }}\left(1+e_{\min }\right)
\end{aligned}
$$

where

$$
f_{1}(e, x):=\frac{1}{e^{2}(1-e x)}\left(4-3 e^{2}-\left(2 e-e^{3}\right) x-\left(3-2 e^{2}\right) x^{2}+e x^{3}\right)
$$

and we used that $f_{1}(e, \cos u)$ is monotone decreasing in the eccentricity $\forall u \in[0,2 \pi]$. Since

$f_{1}(e, x)=\frac{1}{e^{2}(1-e x)}\left(3-2 e^{2}-e x-\left(3-2 e^{2}\right) x^{2}+e x^{3}\right)+\frac{1-e^{2}}{e^{2}}=f_{2}(e, x)+\frac{1-e^{2}}{e^{2}}$

where

$$
f_{2}(e, x):=\frac{\left(1-x^{2}\right)\left(3-2 e^{2}-x\right)}{e^{2}(1-e x)}
$$

Note that, for $|x| \leq 1, f_{2}$ is a positive function which attains maximum for $0 \leq x \leq 1$. For $0 \leq x \leq 1$ the term $1-x^{2}$ is decreasing, while $\left(3-2 e^{2}-x\right) /(1-e x)$ is increasing. A rough estimate of this value is obtained evaluated the first term in $x=0$ and the second one in $x=1$, getting $2(1+e) / e^{2}$. Recollecting we have that

$$
0 \leq f_{1}(e, x) \leq \frac{3+2 e-e^{2}}{e^{2}} \leq \frac{3+2 e_{\min }-e_{\min }^{2}}{e_{\min }^{2}}
$$

Finally it has been used that the function $\sqrt{1-e^{2}}(1-e) / e$ is monotone decreasing. 
By $(42)$

$$
\begin{aligned}
\sup _{\Omega}\left|\partial_{\ell} H\right| \leq & \epsilon M^{\frac{1}{3}} L_{\max }^{2}\left(-1+\frac{1}{\left(1-r_{\max }\right)^{3}}\right) \\
& \cdot \max _{\Omega}\left|\cos g\left(\frac{\sin u}{1-e \cos u}\right)+\sin g\left(\frac{\sqrt{1-e^{2}} \cos u}{1-e \cos u}\right)\right| \\
& +\frac{\epsilon M^{2 / 3} L_{\max }^{4}}{\left(1-r_{\max }\right)^{3}} e_{\max } \\
\leq & \epsilon M^{\frac{1}{3}} L_{\max }^{2}\left(-1+\frac{1}{\left(1-r_{\max }\right)^{3}}\right) \sqrt{\frac{1+e_{\max }}{1-e_{\max }}}+\frac{\epsilon M^{\frac{2}{3}} L_{\max }^{4}}{\left(1-r_{\max }\right)^{3}} e_{\max }
\end{aligned}
$$

where in the last inequality we have used (72) and the fact that

$$
\frac{1+e \cos u}{(1-e \cos u)} \leq \frac{1+e_{\max }}{1-e_{\max }}
$$

Then (56) follows.

We conclude estimating $w$ in (44)

$$
\begin{aligned}
& \sup _{\Omega}|w| \leq\left(\epsilon M^{\frac{1}{3}} L_{\max }\left(-1+\frac{1}{\left(1-r_{\max }\right)^{3}}\right)\right. \\
& \max _{\Omega}\left\{\left|\cos g\left(\frac{\left(1-e^{2}\right) \sin u \cos u}{1-e \cos u}\right)+\sin g \frac{\sqrt{1-e^{2}}}{1-e \cos u}\left(-2 e \cos u+1+\cos ^{2} u\right)\right|\right\} \\
& +\frac{\epsilon M^{\frac{2}{3}} L_{\max }^{3}}{\left(1-r_{\max }\right)^{3}}\left(1-e_{\min }^{2}\right) \\
& \leq \epsilon M^{\frac{1}{3}} L_{\max }\left(-1+\frac{1}{\left(1-r_{\max }\right)^{3}}\right) 2 \sqrt{1-e_{\min }^{2}}+\frac{\epsilon M^{\frac{2}{3}} L_{\max }^{3}}{\left(1-r_{\max }\right)^{3}}\left(1-e_{\min }^{2}\right)
\end{aligned}
$$

where in the last inequality we have used (72) and the fact that

$$
\frac{10-15 e \cos u+6 \cos 2 u-e \cos 3 u}{(1-e \cos u)} \leq 16
$$

Acknowledgments A preliminary version of this paper was contained in the degree thesis of the first author having the last author as supervisor at the University of Roma Tre.

\section{References}

Ambrosetti, A., Prodi, G.: 'A primer of Nonlinear Analysis', Editrice Tecnico Scientifica (1973)

Arnold, V.A., Kozolov, V.V, Neishtadt, A.I.: 'Mathematical aspects of classical and celestial mechanics. (Dynamical systems. III) ', Springer-Verlag, Berlin (2006)

Arnol'd, Vladimir I.; Kozlov, Valery V.; Neishtadt, Anatoly.: ' I. Mathematical aspects of clas- sical and celestial mechanics. Dynamical systems. III.' Encyclopaedia of Mathematical Sciences,Springer-Verlag, Berlin ISBN: 978-3-540-28246-4; 3-540-28246$7(2006)$

Bate, R.R., Mueller,D.D., White,J.E.: 'Foundamentals of Astrodynamics' Dover Publications Ink, New York (1971) 
Belbruno, E.: 'Lunar capture orbits. a method of constructing earth-moon trajectories and the lunar gas mission', Proceedings of AIAA/DGLR/JSASS, pp. 87-1054 (1987)

Belbruno, E.: 'Examples of the Nonlinear Dynamics of Ballistic Capture and Es- cape in the EarthMoon System', AIAA paper, pp. 90-2896 (1990)

Belbruno, E.: 'The Dynamical Mechanism of Ballistic Lunar Capture Transfers in the Four-Body Problem from the Perspective of Invariant Manifolds and Hills Regions', Centre De Recerca Matematica, Barcelona, (1994)

Belbruno, E.: 'Fast Resonance Shifting as a Mechanism of Dynamical Instability Illustrated by Comets and the CHE Trajectories', Annals of the New York Academy of Sciences, Vol. 822, pp.195226 (1997)

Belbruno, E.: 'Analytic Estimation of the WSB and Low Energy Transfers', Contemporary Mathematics, Vol. 292, pp. 17-46 (2002)

Belbruno, E.: 'Capture Dynamics and Chaotic Motions in Celestial Mechanics: With Applications to the Construction of Low Energy Transfers', Princeton University Press (2004)

Belbruno, E., Humble, R.: 'Ballistic Capture Lunar transfer determination for the U.S. Air Force Academy Blue Moon Mission', Advances inAstronautical Sicence, Spaceflight Mechanics (1997)

Belbruno, E., Miller, J.: 'Sun-perturbed earth-to-moon transfers with a ballistic capture', Journal of Guidance, Control, and Dynamics, vol. 16, n.4, pp.770-775 (1993)

Bello-Mora, M., Graziani, F., Teofilatto, P., Circi, C., Porfilio, M., Hechler, M.: 'A Systematic Analysis on Weak Stability Boundary Transfers to the Moon', Paper IAF-00-A.6.03, Proceedings of the International Astronautical Conference (2000)

Carrico, J.P., Belbruno, E.: 'Calculation of weak stability boundary ballistic lunar transfer trajectories', American Institute of Aeronautics and Astronautics (2006)

Celletti, A.; Chierchia, L.: ' KAM stability and celestial mechanics.' Mem. Amer. Math. Soc. 187 , no. $878(2007)$

Di Gregorio, L.: 'Delaunay-poincarè coordinates close to collinear lagrangian point of the PRC3BP', Institut fur Industremathik University of Paderborn (2008)

Diacu, F.: 'The solution of the n-body Problem', The Mathematical Intelligencer Vol.18, N.3, pp. 6670 (1996)

Gascheau, M.: 'Examen d'une classe d'equations differentielles et application a un cas particulier du probleme des trois corps', Compt. Rend. 16, Princeton (1843)

García, F., Gómez, G.: 'A note on weak stability boundaries', Celest. Mech. Dyn. Astr., Vol.97, pp. 87-10 (2007)

Goldstein, Poole, Safko: 'Classical Mechanics', Addison Wesley; 3 edition ISBN-13: 978020165702(2001)

Gómez, G., Jorba, A., Masdemont, J. J., Simó, C: 'Study of the Transfer from the Earth to a Halo Orbit Around the Equilibrium Point $L_{1}$ ', Celest. Mech. Dyn. Astr., Vol.56, N.4 pp. 541-562 (1993)

Gómez, G., Koon, W. S., Lo, M. W., Marsden, J. E., Masdemont, J., Ross, S. D.: ' Connecting orbits and invariant manifolds in the spatial restricted three-body problem.', Nonlinearity, Vol.17 (5), pp. 1571-1606 (2004)

Koon, W.S., Lo, M.W., Marsden, J.E., Ross, S.D.: 'Heteroclynic connections between periodic orbits and resonance transitions in celestial mechanics', Preprint (1999)

Koon, W.S., Lo, M.W., Marsden, J.E. , Ross, S.D.: 'Low Energy Transfer to the Moon ', Celest. Mech. Dyn. Astr., Vol.81, pp. 63-73 (2001)

Koon, W.S., Lo, M., Marsden, J.E., Ross., S.D.: 'Dynamical Systems, the Three-Body Problem and Space Mission Design', pp. 123-130. Marsden Books, London (2008) 
Mingotti, G., Topputo, F., and Bernelli-Zazzera, F. : 'Low-energy, low-thrust transfers to the Moon', Celest. Mech. Dyn. Astr., Vol.105, pp. 61-74 (2009)

Poincaré, H.: 'Les methodes nouvelles de la mecanique celeste', Gauthier-Villars et fils (1899)

Romagnoli, D., Circi, C.: 'Earth.Moon Weak Stability Boundaries in the restricted three and four body problem', Celest. Mech. Dyn. Astr., Vol.103, pp. 79-103 (2009)

Stiefel, E. L.; Scheifele, G.: 'Linear and regular celestial mechanics. Perturbed two-body motion, numerical methods, canonical theory.', Die Grundlehren der mathematischen Wissenschaften, Band 174. Springer-Verlag, New York-Heidelberg,(1971)

Topputo, F.: 'Low-Thrust Non-Keplerian Orbits: Analysis, Design, and Control', PhD Thesis, Politecnico di Milano (2007)

Topputo, F., Belbruno, E.: 'Computation of weak stability boundaries: Sun.Jupiter system', Celest. Mech. Dyn. Astr., Vol.105, pp. 3-17 (2009)

Topputo, F., Belbruno, E., Gidea,M.: 'Resonant motion, ballistic escape, and their applications in astrodynamics', Science Direct (2008) 